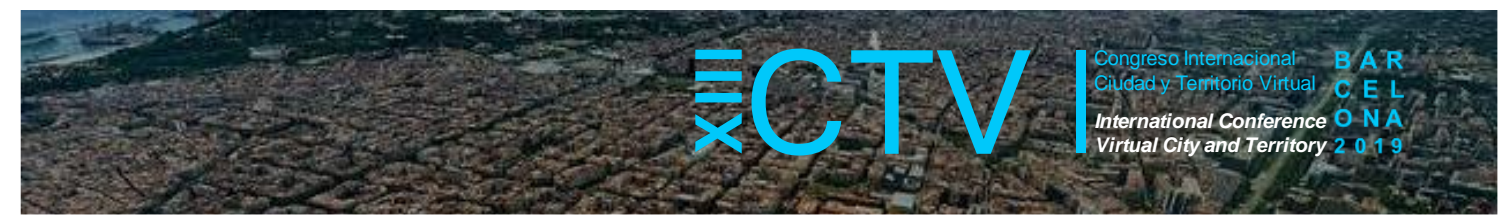

\title{
LA COMPOSICIÓN DE LOS ESPACIOS VERDES Y NIVELES DE ACTIVIDAD FÍSICA DE LA GENTE MAYOR EN BARCELONA
}

\author{
Vich, Guillem ${ }^{1}$; Dopico, Javier ${ }^{2 *}$; Miralles-Guasch, Carme ${ }^{3}$
}

Remisión inicial: 2019-06-14; Remisión definitiva: 2019-09-29; Publicación: 2019-12-21

Citación: Vich G. et al. (2019). La composición de los espacios verdes y niveles de actividad física de la gente mayor en Barcelona. En XIII CTV 2019 Proceedings: XIII International Conference on Virtual City and Territory: "Challenges and paradigms of the contemporary city": UPC, Barcelona, October 2-4, 2019. Barcelona: CPSV, 2019, p. 8555. E-ISSN 2604-6512. DOI http://dx.doi.org/10.5821/ctv.8555

\section{Resumen}

El estudio de los espacios verdes urbanos (UGS en inglés) ha recibido una gran atención tanto de los académicos como de los planificadores urbanos, ya que ofrecen una gama de servicios suponiendo una mejora de los valores para la sociedad. Los espacios abiertos urbanos provistos de elementos naturales se han relacionado con una serie de beneficios, tanto para el medio ambiente y la salud como para el bienestar de los residentes urbanos. Esto es posible ya que estos lugares ofrecen a las personas la posibilidad de interactuar socialmente además de con el medio ambiente, en la medida en que promueven actividades de ocio y recreación al aire libre. Son, por lo tanto, lugares adecuados para la práctica de actividad física. Los beneficios de salud fisiológicos y psicosociales de los UGS son importantes para abordar los desafíos futuros que implica el envejecimiento de las sociedades modernas. La asociación entre los visitantes de estos espacios y los aumentos en los niveles de AF está relacionada con una amplia serie de beneficios especialmente entre los ancianos. La forma y la composición de los UGS pueden variar ampliamente desde el punto de vista de la infraestructura proporcionada. A su vez, estas características pueden influir en el uso que hacen las personas en estos espacios. Es escasa la evidencia existente a la hora de explorar la relación entre la provisión de diferentes tipos de paisajes naturales presentes en los UGS, así como los diferentes tipos de superficies disponibles. A través de la categorización de los paisajes naturales esta exploración es posible. Para contribuir en este campo de la investigación, esta investigación tiene como objetivo analizar la relación entre la composición de UGS y su uso por parte de personas mayores en una ciudad mediterránea como Barcelona. Más específicamente, este estudio explora las relaciones entre la provisión de diferentes tipos de paisajes naturales y los tipos de superficies transitables disponibles en UGS con el tiempo empleado y la intensidad de la AF de las actividades realizadas por las personas mayores. Adicionalmente, también se analiza el efecto de ciertas características individuales tales como el género o la edad. Para llevar a cabo este estudio, fue precisa la utilización de datos de seguimiento GPS en combinación con datos de acelerómetro registrados en 63 adultos mayores que residen en Barcelona. Se proporcionó a los participantes dispositivos capaces de realizar estos registros que debieron usar durante 7 días consecutivos con el fin de obtener sus ubicaciones diarias y la intensidad de la AF. Se calculó así el tiempo total que pasaron dentro de estos espacios además de la intensidad (sedentaria o activa) de sus actividades para explorar el uso de las diferentes áreas según el tipo de vegetación y la diversidad del paisaje además del tipo de suelo. Los resultados de este estudio han mostrado que los participantes pasaron una mediana de 8,5 minutos dentro de los UGS analizados, y que las actividades sedentarias registradas predominan sobre las activas. Se ha demostrado que las superficies duras están positivamente vinculadas con el tiempo total empleado, mientras que existe una asociación negativa entre las superficies blandas y el tiempo dedicado a un comportamiento activo. Las principales conclusiones extraídas del presente trabajo indican que la edad o la salud de la población son aspectos clave para tener en cuenta a la hora de construir y diseñar UGS que sean inclusivos y accesibles para todos los perfiles de la sociedad, destacando también la importancia de otros aspectos tales como la accesibilidad (en cuanto a la distancia desde el hogar). Asimismo, el tipo de superficie transitable juega un papel relevante. Las superficies duras se relacionan con un mayor tiempo total en estos espacios mientras que las superficies blandas implican un menor tiempo dedicado a la AF de comportamiento activo.

\footnotetext{
${ }_{1}^{1}$ Departament de Geografia. Universitat Autònoma de Barcelona, https://orcid.org/0000-0002-6419-1913; ${ }^{2}$ Institut de Ciència i Tegnologia Ambientals (ICTA). Universitat Autònoma de Barcelona, ${ }^{3}$ Departament de Geografia y Institut de Ciència i Tegnologia Ambientals (ICTA). Universitat Autònoma de Barcelona, https://orcid.org/0000-0003-4821-9776. *Correo de contacto: javier.dopico@e-campus.uab.cat
} 


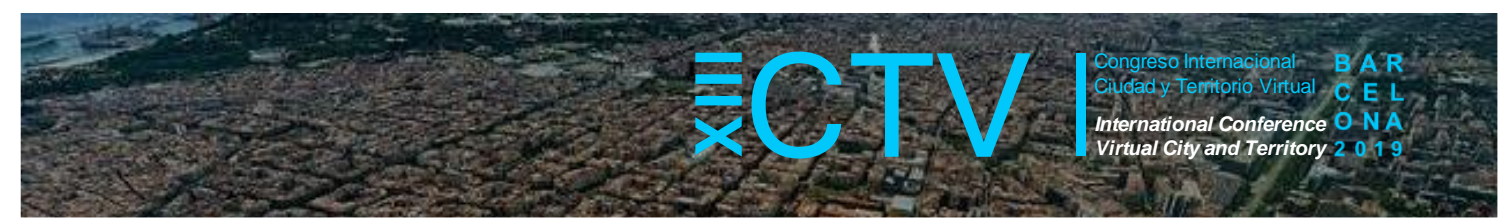

\section{Abstract}

The study of urban green spaces (UGS) has received great attention from both academics and urban planners, as they offer a broad range of services offering an improvement in values for society. Urban open spaces provided with natural elements have been linked to a series of benefits, both for the environment and health and for the well-being of urban residents. This is possible since these places offer to the people the possibility to interact socially as well as with the environment, to the extent that they promote outdoor leisure and recreation activities. They are, therefore, suitable places for the practice of physical activity. The physiological and psychosocial health benefits of UGS are important to address the future challenges of aging in modern societies. The association between visitors to these spaces and increases in PA levels is related to a wide range of benefits especially among the elderly. The shape and composition of UGS can vary widely from the point of view of the infrastructure provided. In turn, these characteristics can influence the use that people make in these spaces. There is little evidence to explore the relationship between the provision of different types of natural landscapes present in UGS as well as the different types of surfaces available. Through the categorization of natural landscapes this exploration is possible. To contribute to this field of research, this research aims to analyze the relationship between the composition of UGS and its use by older people in a Mediterranean city like Barcelona. More specifically, this study explores the relationships between the provision of different types of natural landscapes and different types of available surfaces in UGS with the time spent and the PA intensity of the performed activities by the elderly. Additionally, the effect of certain individual characteristics such as gender or age is also analyzed. To carry out this study, it was necessary the use of GPS tracking data in combination with accelerometer data recorded in 63 older adults residing in Barcelona. Participants were provided of devices capable of making these records. They had to use it for 7 consecutive days in order to obtain their daily locations and the intensity of PA. Thus, the total time spent within these spaces was calculated in addition to the intensity (sedentary or active) of their activities to explore the use of different areas according to the type of vegetation and natural landscape in addition to the type of soil. The results of this study have shown that participants spent a median of 8.5 minutes within the analyzed UGS, and that registered sedentary activities predominate over active ones. It has been shown that hard soils are positively linked to the total time spent, while there is a negative association between soft soils and time spent on active behavior. The main conclusions drawn from this work indicate that age and health of the population are key aspects to take into account when building and designing UGS that are inclusive and accessible to all profiles of society, also highlighting the importance of other aspects such as accessibility (in terms of distance from home). Also, the type of surface plays a relevant role. Hard soils are related to a greater total time in these spaces while soft soils involve less time devoted to PA of active behavior.

Palabras Clave: Espacios verdes urbanos; actividad física, tercera edad, Barcelona

Key words: Green urban spaces; physical activity; elderly; Barcelona

\section{Introducción}

En un contexto global de rápida urbanización, la provisión de Espacios Verdes Urbanos (UGS) se ha señalado como una estrategia clave para los responsables políticos para el logro del desarrollo urbano sostenible, pero también para la mejora de la salud y el bienestar de los residentes urbanos (Kabisch et al., 2016; WHO 2016). Además de los muchos beneficios para la salud, estar en contacto con la naturaleza en espacios verdes ha demostrado disminuir el estrés fisiológico (Roe et al., 2013), impulsar las interacciones sociales (Chiang y Li, 2019) y mitigar la contaminación del aire, así como los niveles de calor y ruido (Livesley, McPherson y Calfapietra, 2016; Margaritis y Kang, 2017). Estos beneficios son posibles al ofrecer a las personas la posibilidad de interactuar en la medida que se promueven actividades de ocio y recreación al aire libre, siendo por lo tanto los UGS lugares adecuados para la práctica de actividad física (AF) (Vich, Marquet \& Miralles, 2019a). Los beneficios para la salud física y mental vinculados a los UGS también son clave para abordar los desafíos en relación con el envejecimiento de las actuales sociedades modernas (Barnett, Barnett, Nathan, Van Cauwenberg y Cerin, 2017). La posibilidad de realizar actividad física en estos espacios se ha asociado con disminuciones en la incidencia de enfermedades cardiovasculares y crónicas y de morbilidad, pero también con la mejora de la capacidad funcional y la cognición entre la población de mayor edad (Heiland et al., 2019; Recchioni et al., 2017). 


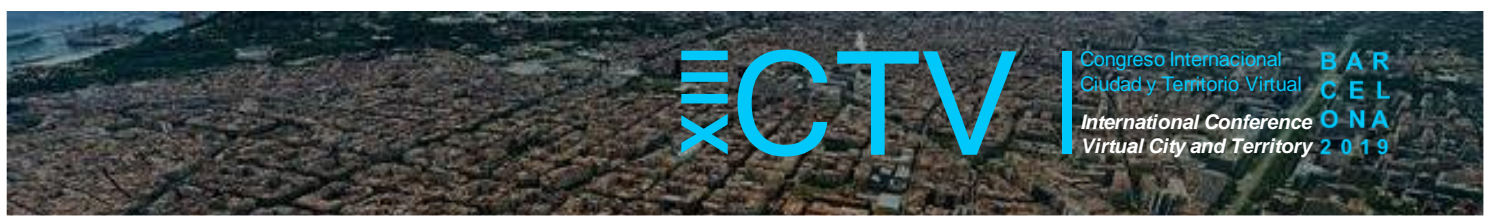

Las características y composición de los UGS pueden variar ampliamente según la diversidad del paisaje y la infraestructura disponible, lo que puede influir en el tipo de actividades que se realicen, así como en los beneficios derivados. Los elementos de la infraestructura, como los parques infantiles o los campos deportivos, se han relacionado ampliamente con el aumento de los niveles de AF por parte de los visitantes de UGS (Wu \& Song, 2017). Sin embargo, existe una menor evidencia con respecto a otros elementos comúnmente disponibles en UGS, como el tipo de caminos y superficies transitables en estos espacios, que son de gran importancia para los visitantes mayores (Gaikwad y Shinde, 2019). Además, teniendo en cuenta que la función principal de UGS es ofrecer a los visitantes la posibilidad de estar rodeados de vegetación y ver elementos naturales (Wang, Zhao, Meitner, Hu y Xu, 2019), pocos estudios se han centrado en el vínculo entre la diversidad del paisaje y las actividades realizadas en estos espacios en función de su intensidad física (Daniels et al., 2018).

De este modo, el objetivo de este estudio es analizar la relación entre las características del paisaje y la infraestructura para caminar disponible con el tiempo que se pasa en UGS y el tipo de actividades realizadas por adultos mayores. Para hacerlo, este estudio utiliza datos de seguimiento GPS en combinación con datos de acelerómetro para obtener las ubicaciones geolocalizadas y la intensidad de la actividad física realizada dentro de un conjunto de UGS de la ciudad de Barcelona.

\section{Marco teórico}

La diversidad de la vegetación y los elementos naturales presentes en los espacios verdes urbanos (UGS) constituyen su paisaje, lo que hace que estos espacios sean más o menos atractivos para ser visitados y realizar ciertas actividades. Así, las áreas abiertas con vegetación, como las praderas, actúan como lugares recreativos donde se pueden desarrollar interacciones sociales o actividades deportivas (Arnberger y Eder, 2011; Zhai, Baran y Wu, 2018). Del mismo modo, la provisión de áreas sombreadas por los árboles supone un impacto positivo al incentivar el caminar o sentarse por éstas (Wang et al., 2019). Asimismo, las áreas cubiertas de flores suponen una mejorar en el atractivo de los UGS además de alentar a caminar alrededor de ellas (Hoyle et al., 2017). En esta misma línea, la presencia de elementos acuáticos como lagos o estanques ha demostrado promover la realización de actividades sedentarias mientras se visualizan estos elementos a su alrededor (Marquet et al., 2019; White et al., 2010). Además, los arbustos, especialmente los de baja altura, son otro tipo de elementos del paisaje asociado percibido positivamente, ya que han demostrado reducir la percepción de inseguridad en los UGS (Lindgren y Nielsen, 2012).

La calidad y cantidad de las instalaciones y servicios también son características importantes que permiten realizar diferentes tipos de actividades (Bauman et al., 2012). La presencia de carriles bici, parques infantiles y campos deportivos son elementos comunes en los que los visitantes pueden estar físicamente activos (Baran et al., 2014), mientras que las áreas de picnic y barbacoa están vinculadas a comportamientos más sedentarios (Jansen, Ettema, Kamphuis, Pierik y Dijst, 2017; Shores y West, 2006). Además, la presencia de fuentes potables, papeleras y una buena red de iluminación son también elementos relevantes que incrementan las visitas a UGS (Veitch et al., 2017). Respecto a las personas mayores, los baños y bancos son elementos clave para fomentar la actividad física y las actividades sedentarias (Arnberger y Eder, 2015). Sin embargo, una infraestructura crucial y en general 


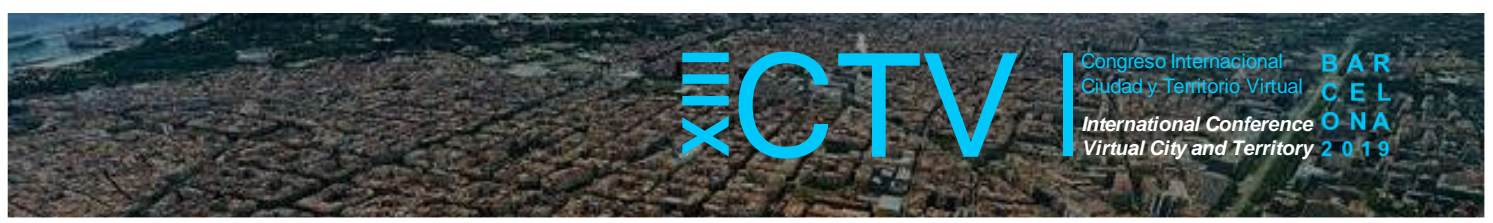

poco estudiada, especialmente relacionada con los adultos mayores, son los caminos y diferentes tipos de superficies transitables. Las rutas bien definidas y de largo recorrido son infraestructuras básicas en los UGS ya que intensifican los niveles de actividad física entre los visitantes mayores en comparación con otras áreas como las praderas (Besenyi, Kaczynski, Stanis y Vaughan, 2013). Se ha comprobado que los caminos duros y pavimentados fomentan actividades como caminar, andar en bicicleta o pasear perros (Arnberger \& Eder, 2015; Schipperijn et al., 2013). Las personas mayores, ya que estos son más sensibles a las superficies inestables, prefieren utilizar suelos pavimentados debido a su mayor estabilidad y percepción de seguridad (Rodiek y Fried, 2005). En esta línea, el estado en el que se mantiene esta infraestructura también es de gran importancia para mejorar la experiencia del visitante (Zhai \& Baran, 2017).

\section{Metodología}

\section{1. Área de estudio}

Barcelona es una ciudad costera mediterránea española ubicada en el noreste de la Península Ibérica ( $41^{\circ} 23$ '02' 'N $02{ }^{\circ} 07$ ' 59 " E). Con una superficie de $102,16 \mathrm{~km}^{2}$, se encuentra entre la confluencia de los ríos Llobregat y Besòs al suroeste y noreste, respectivamente, y al este del Parque Natural de Collserola (Ajuntament de Barcelona, 2018a).

La ciudad cuenta con 1.135 ha de espacios verdes urbanos (UGS) en forma de parques y jardines, lo que equivale a $7,1 \mathrm{~m}^{2}$ de espacio verde por habitante (Ajuntament de Barcelona, 2018b). Sin embargo, esta es una proporción menor en comparación con otras ciudades europeas con cifras que alcanzan los $300 \mathrm{~m} 2$ por habitante en algunos casos, especialmente en el norte del continente (Fuller y Gaston, 2009). Un 22,6\% de la superficie vegetal de la ciudad es autóctona, con predominio de Quercus ilex, Pinus halapensis y Platanus $x$ acerifolia, que representan el $49 \%$ de todos los árboles urbanos de la ciudad (Ajuntament de Barcelona, 2018c).

\subsection{Participantes y diseño del estudio}

Se obtuvieron los patrones diarios de actividad física de 269 participantes, reclutados de diferentes centros para personas mayores de la ciudad entre junio de 2016 y junio de 2017. Se contactó con todos los centros para personas mayores de Barcelona, tanto públicos $(N=21)$ como fundaciones privadas $(\mathrm{N}=15)$, de los cuales casi la mitad accedió para participar $(\mathrm{N}=$ 14). Se trató de equilibrar la muestra de estos centros entre los ubicados en barrios de rentas altas y los de rentas bajas. Tras explicar las condiciones de participación en cada uno de los centros, se buscó una participación equitativa de género, resultando finalmente en 153 mujeres por 116 participantes varones. De los 269 participantes iniciales, 122 de ellos eran residentes en la ciudad de Barcelona. Finalmente, 63 de ellos fueron seleccionados para los análisis ya que visitaron al menos un UGS durante el estudio. Se pidió a los participantes interesados que buscaran voluntarios adicionales en su círculo social que no asistieron a los centros para personas mayores.

Posteriormente, distribuyendo un documento informativo sobre el proyecto y firmando un formulario de consentimiento informado, a los participantes se les proporcionó un acelerómetro 


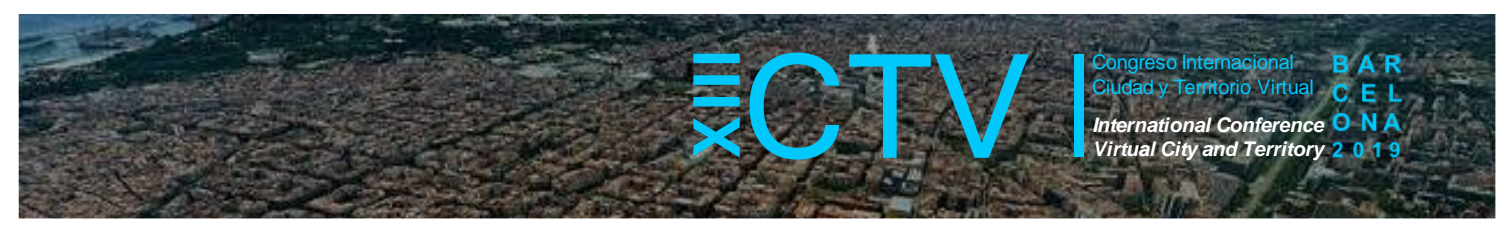

para usar en la muñeca junto con un dispositivo GPS que deberían utilizar durante 7 días consecutivos para obtener sus niveles actividad física y ubicaciones diarias respectivamente. Además, se les pidió a los participantes que rellenaran un cuestionario sobre su perfil sociodemográfico, la movilidad diaria y los hábitos de actividad física, así como su percepción sobre su vecindario. El estudio recibió la aprobación de la Comisión de Ética en la Experimentación Animal y Humana de la UAB (CEEAH-3656).

\subsection{Fuentes de datos y medición}

Para el propósito de este estudio, los datos de seguimiento y actividad física proporcionados por dispositivos GPS y acelerómetros se combinaron con datos SIG disponibles públicamente por parte del Ayuntamiento de Barcelona para extraer el uso de UGS en Barcelona por parte de sus residentes mayores.

\subsubsection{Datos SIG}

En primer lugar, se extrajeron todos los polígonos del Mapa de Uso del Suelo de Barcelona (Ajuntament de Barcelona, 2016) categorizados como "Parques y Jardines" (Ver Figura 1). En segundo lugar, todos estos espacios fueron visitados y valorados para verificar la precisión de los límites y dividirlos y clasificar las áreas interiores según la diversidad del paisaje y el tipo de superficie transitable. En tercer lugar, dado que la planificación y construcción de algunos espacios no estaba completa y, en otros casos, los límites no estaban representados con precisión, fue necesario volver a dibujar el polígono para hacerlo lo más similar posible a la realidad. Cuarto, se fusionaron los polígonos resultantes que estaban a menos de 10 metros de distancia. Quinto, finalmente se seleccionaron los de más de 1 hectárea (ver Figura 1).

Aunque no existe un acuerdo general sobre un umbral de tamaño crítico para beneficios de salud específicos (WHO 2016), el umbral seleccionado de 1 hectárea se ajusta a los límites utilizados de 0.5 y $2 \mathrm{HA}$ considerados por las recomendaciones de salud internacionales y nacionales (Ambiente Italia Research Institute 2003; Natural England 2010).

Después de visitar y valorar los 122 UGS restantes del Mapa de Uso del Suelo, las áreas dentro de estos espacios se clasificaron según la diversidad del paisaje y el tipo de superficie transitable (ver Figura 2). Los espacios con elementos naturales con UGS se identificaron como áreas de bosques, matorrales o praderas. De dichas áreas, aquellas con superficies transitables se dividieron en superficies duras, mixtas o blandas. Los elementos del agua también fueron identificados y clasificados.

Finalmente, de los UGS seleccionados y clasificados del Mapa de Uso del Suelo de Barcelona, se seleccionaron aquellos polígonos que incluían puntos de seguimiento GPS de los participantes dentro de sus límites, lo que representa un $83 \%$ de los UGS visitados y clasificados. Como se puede ver en la Tabla 1, el bosque es el tipo de paisaje con mayor representación con un $47.2 \%$ del total, seguido de superficies duras con un $16,4 \%$ y mixtas con un $14 \%$. Por último, los arbustos y praderas (6.7 y 6.8 respectivamente), y el agua (4.4\%) y la superficie blanda (4.3\%) fueron las áreas menos disponibles. 


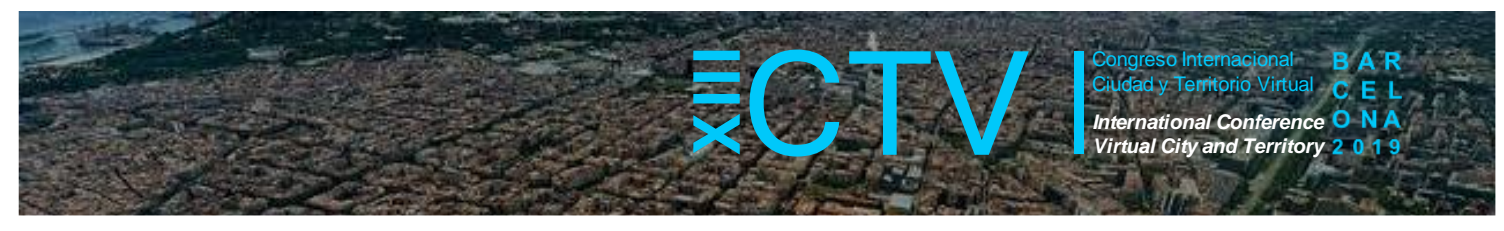

Figura 1. Distribución de los UGS disponibles en la ciudad de Barcelona y la muestra analizada

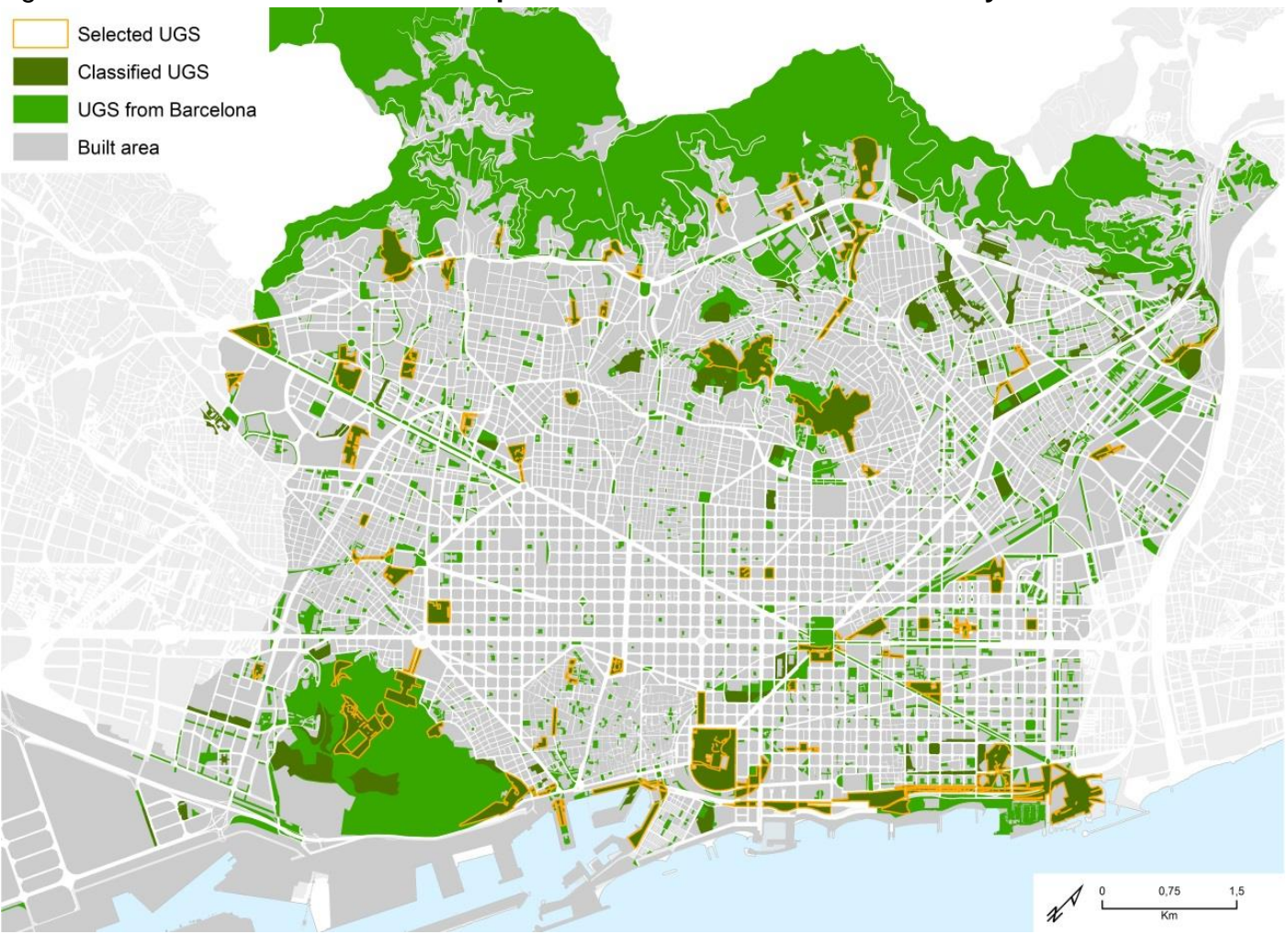

Fuente: producción propia

Figura 2. Ejemplo de caracterización de UGS por tipo de vegetación y superficie transitable

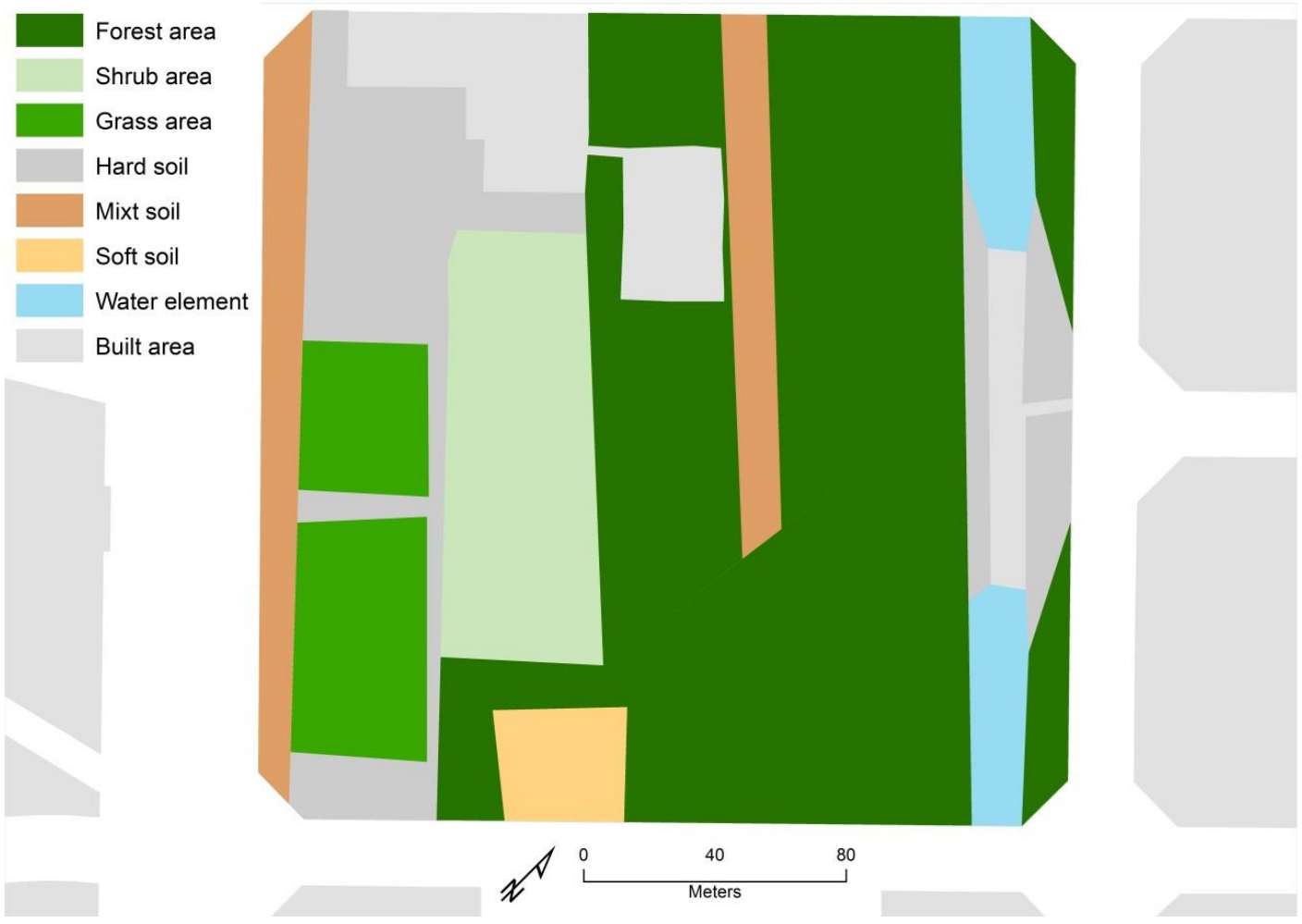

Fuente: producción propia 


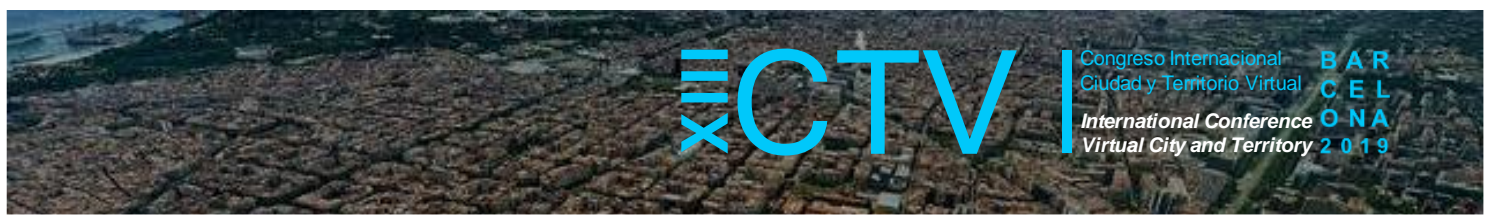

\subsubsection{Datos de seguimiento y acelerómetro}

Los datos de seguimiento recopilados reflejaban, por un lado, la posición de los participantes en intervalos de 15 gracias al uso de registros GPS (Qstarz Q-1000XT). Los datos del acelerómetro se recopilaron utilizando dispositivos ActiGraph GT3X + y para el presente estudio se clasificaron en dos categorías de intensidad de AF: "sedentaria" y "activa". Siguiendo a Esliger et al. (2011), se usó un umbral de <216 recuentos de VM por minuto para definir el tiempo sedentario, donde más de 216 recuentos de VM se clasificaron como "activos". Los datos de GPS y acelerómetro se fusionaron utilizando el software PALMS (Jankowska, Schipperijn y Kerr, 2015) para acoplar información espacial, temporal y relacionada con la AF.

\subsubsection{Medición del uso de UGS}

Los participantes de la muestra registraron una base de datos inicial de 5.423 .327 puntos de teleseguimiento o tracking en el proceso de recopilación de datos. Este número se redujo a 33,260 al eliminar todos los puntos fuera de los polígonos seleccionados de la capa SIG modificada del Mapa de Uso del Suelo (Ver Figura 3). La base de datos se redujo nuevamente a 14.323 puntos de seguimiento después de seleccionar a los participantes que visitaron UGS dentro de un umbral temporal mínimo de al menos 3 minutos consecutivos en el mismo (Stewart et al., 2016). Finalmente, esos puntos de seguimiento registrados en el agua fueron eliminados ya que no son instalaciones para nadar, por lo tanto, dichos puntos registrados en elementos acuáticos podrían deberse a errores de precisión del GPS. La base de datos final acumuló 14,227 puntos de seguimiento, que representaron 290 visitas a 61 UGS diferentes por parte de 63 usuarios.

Tabla 1. Características de los espacios verdes urbanos analizados

\begin{tabular}{|c|c|c|c|c|c|c|}
\hline \multirow[t]{2}{*}{ Clasificación } & \multirow[t]{2}{*}{ Área } & \multirow[t]{2}{*}{ Definición } & \multicolumn{2}{|c|}{ UGS analizados } & \multicolumn{2}{|c|}{ UGS con puntos GPS } \\
\hline & & & $\mathrm{m}^{2}$ & $\%$ & $\mathrm{~m}^{2}$ & $\%$ \\
\hline \multirow[t]{3}{*}{$\begin{array}{l}\text { Diversidad de } \\
\text { paisaje }\end{array}$} & Bosque & $\begin{array}{l}\text { Área ocupada } \\
\text { mayoritariamente } \\
\text { por árboles }\end{array}$ & $3,282,159$ & 63.5 & 29,046 & 47.2 \\
\hline & Arbusto & $\begin{array}{l}\text { Área ocupada } \\
\text { mayoritariamente } \\
\text { por arbustos }\end{array}$ & 215,230 & 4.2 & 4,192 & 6.8 \\
\hline & Pradera & $\begin{array}{l}\text { Área ocupada } \\
\text { mayoritariamente } \\
\text { por praderas }\end{array}$ & 878,604 & 17.0 & 4,139 & 6.7 \\
\hline \multirow{3}{*}{$\begin{array}{c}\text { Tipo de } \\
\text { superficie }\end{array}$} & $\begin{array}{l}\text { Superficie } \\
\text { dura }\end{array}$ & $\begin{array}{l}\text { Suelo compuesto } \\
\text { por cemento o } \\
\text { azulejo }\end{array}$ & 362,266 & 7.0 & 10,099 & 16.4 \\
\hline & $\begin{array}{l}\text { Superficie } \\
\text { blanda }\end{array}$ & $\begin{array}{c}\text { Suelo compuesto } \\
\text { por gravilla }\end{array}$ & 142,539 & 2.8 & 8,625 & 14.0 \\
\hline & $\begin{array}{l}\text { Superficie } \\
\text { mixta }\end{array}$ & $\begin{array}{c}\text { Áreas complejas con } \\
\text { diferentes tipos de } \\
\text { suelo }\end{array}$ & 174,971 & 3.4 & 2,651 & 4.3 \\
\hline Otros & Agua & $\begin{array}{l}\text { Cuerpo de agua tal } \\
\text { como Fuente o } \\
\text { estanque }\end{array}$ & 108,940 & 2.1 & 2,723 & 4.4 \\
\hline $\begin{array}{l}\text { Superficie } \\
\text { total }\end{array}$ & & & $5,164,710$ & 100.0 & 61,475 & 100.0 \\
\hline
\end{tabular}




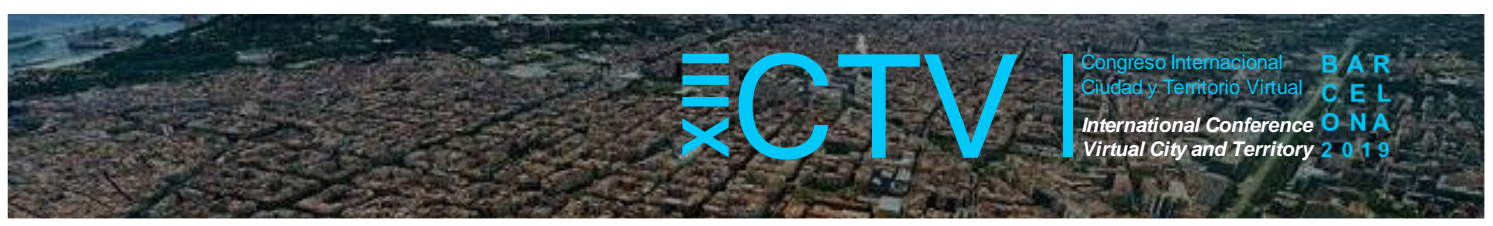

\subsection{Muestra}

La muestra final incluye 63 usuarios de entre 65 y 92 años. Los participantes masculinos estuvieron más presentes que las mujeres (55.6\% vs $44.4 \%$ respectivamente), así como la proporción de participantes más jóvenes (54\%) fue mayor que la de los de mayor edad (46\%).

\subsection{Análisis de datos}

Para analizar el uso de los diferentes UGS, la variable principal es el tiempo pasado en cada área. El análisis explora además el tiempo dedicado a cada tipo de paisaje por intensidad de actividad física. Debido a la distribución asimétrica y no normal de la muestra, se usó la mediana para informar sobre el tiempo promedio de permanencia en cada área de UGS.

Figura 3. Ejemplo de rastreo GPS de un participante en uno de los UGS seleccionados

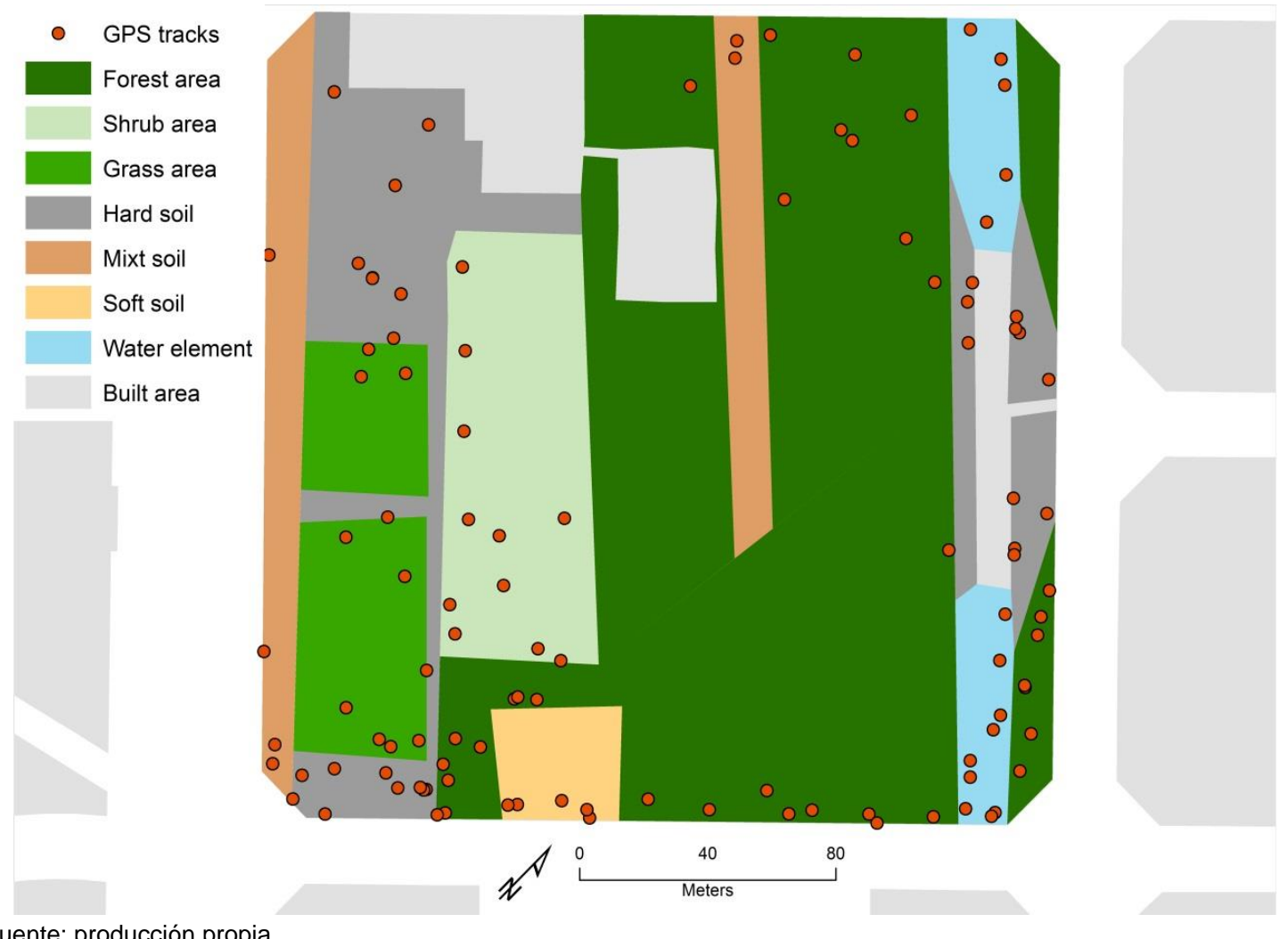

\section{Resultados}

La Tabla 2 resume el tiempo dedicado a cada tipo de área y de superficie en base a la intensidad de la actividad física y el tiempo empleado. Los resultados muestran una fuerte relación entre la proporción del tipo de área y el tiempo que se pasa en ellas. Sin embargo, existen diferencias al comparar los porcentajes del tipo de área con el porcentaje del tiempo pasado en cada una. El paisaje predominante se corresponde con el bosque (29,046 m2 y $47.2 \%$ de la superficie total). Resultó ser además el área donde los participantes pasaron el mayor porcentaje del tiempo total $(41.4 \%)$. Sin embargo, la proporción del tiempo total en el bosque disminuye ligeramente atendiendo al tipo de actividad física y su intensidad $(38.0 \%$ en 


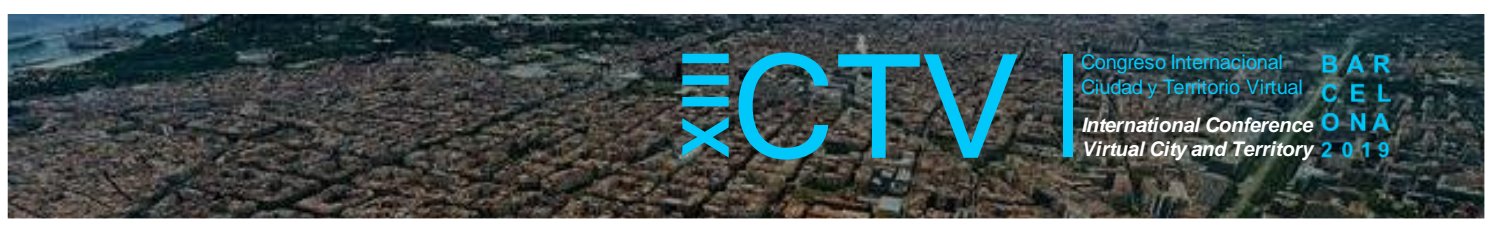

sedentario y $41 \%$ activo). También se ha detectado una diferencia notable en el caso de superficies duras, aunque obtuvo la segunda mayor proporción de tiempo empleado en los UGS seleccionados (22.9\%). Esto resultó ser considerablemente más alto que la proporción de este tipo de superficie (16.4\%). Esta tendencia también se confirma para actividades físicamente intensas y es aún mayor para actividades sedentarias. Los participantes también registraron una mayor proporción de tiempo para actividades de tipo sedentario en superficies blandas ( $10.0 \%$ del tiempo total, $11.1 \%$ del tiempo sedentario y $10.7 \%$ del tiempo activo) y en zonas arbustivas $(9.4 \%, 9.8 \%$ y $8.5 \%$ respectivamente) que la proporción disponible de estas áreas en los UGS analizados. Por otro lado, el tiempo total y el tiempo de actividades sedentarias y activas registradas en las praderas $(2.8 \%, 2.4 \%$ y $2.7 \%$ respectivamente) fueron menores que la proporción de este tipo de área (6.7\%). En cuanto al uso de superficies mixtas, los registros fueron más elevados $(13.6 \%, 14.2 \%$ y $14.9 \%$ respectivamente) que su disponibilidad (4.3\%).

Tabla 2. Estadísticas descriptivas del uso de diferentes áreas dentro de UGS por tiempo e intensidad

\begin{tabular}{ccccccccc}
\hline & \multicolumn{2}{c}{ Área } & \multicolumn{2}{c}{ Tiempo total } & \multicolumn{2}{c}{ Tiempo sedentario } & \multicolumn{2}{c}{ Tiempo activo } \\
\hline Categoría & $\mathbf{m}^{\mathbf{2}}$ & $\%$ & Min & $\%$ & Min & $\%$ & Min & $\%$ \\
\hline Bosque & 29,046 & 47.2 & 4.8 & 41.4 & 2,8 & 38.0 & 2.0 & 41.0 \\
\hline Arbusto & 4,192 & 6.8 & 5.3 & 9.4 & 4.5 & 9.8 & 1.7 & 8.5 \\
\hline Pradera & 4,139 & 6.7 & 1.1 & 2.8 & 1.8 & 2.4 & 0.5 & 2.7 \\
\hline $\begin{array}{c}\text { Superficie } \\
\text { dura }\end{array}$ & 10,099 & 16.4 & 3.0 & 22.9 & 2.1 & 24.5 & 1,5 & 22.2 \\
\hline $\begin{array}{c}\text { Superficie } \\
\text { Mixta }\end{array}$ & 2,651 & 4.3 & 4.1 & 13.6 & 3.3 & 14.2 & 2.3 & 14.9 \\
\hline $\begin{array}{c}\text { Superficie } \\
\text { blanda }\end{array}$ & 8,625 & 14.0 & 3.5 & 10.0 & 3.2 & 11.1 & 1.4 & 10.7 \\
\hline Total & 58,752 & 100.0 & 8.5 & 100.0 & 6.5 & 100.0 & 3.5 & 100.0 \\
\hline
\end{tabular}

\section{Discusión}

Este estudio ha explorado la relación entre la provisión de diferentes tipos de paisajes con vegetación y superficies transitables con la intensidad de las actividades realizadas en los espacios verdes urbanos (UGS) por un grupo de adultos mayores residentes en Barcelona. En general, se ha mostrado que los participantes pasaron períodos de tiempo cortos dentro de los UGS analizados, primando el comportamiento sedentario sobre otras actividades más intensas. El bosque ha sido la categoría de estos espacios donde los participantes pasaron la mayor parte del tiempo, tanto del total como del sedentario y activo, mientras que la superficie dura resultó ser el tipo de superficie transitable más utilizada.

Atendiendo a las características de los UGS analizados, cabe resaltar la importancia de las superficies transitables. Por un lado, la mayor presencia de superficies duras se ha asociado con pasar más tiempo en el parque, además del gran uso de las superficies mixtas. Por otro lado, las superficies blandas se vinculan con menores proporciones de uso pese a su disponibilidad. Asimismo, senderos de fácil caminabilidad y sin obstáculos alientan las actividades al aire libre entre las personas mayores y registran a su vez los niveles más altos de actividad física dentro de UGS (Sugiyama y Thompson, 2008). Se podría por lo tanto secundar así la importancia de las superficies duras entre los usuarios mayores sobre otro tipo de suelo o ambiente cuando se trata de pasar más tiempo en UGS. La presencia de diferentes tipos de vegetación en el paisaje se evidencia como un factor no relevante en cuanto al tiempo 


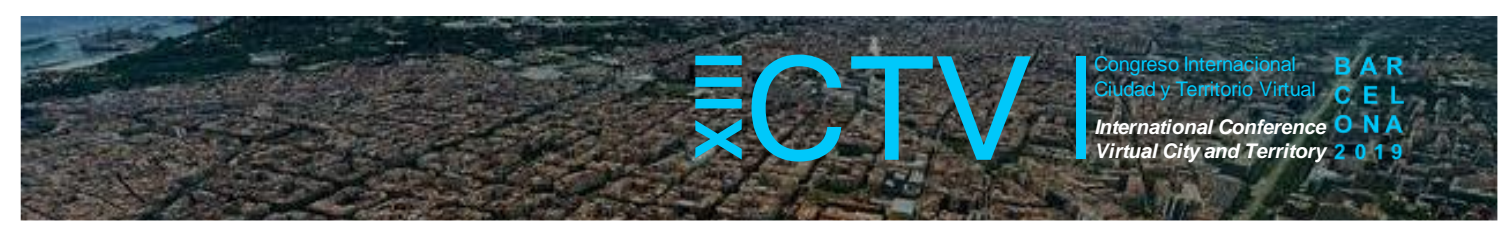

total, sedentario y en activo, lo cual también ha sido confirmado por otros estudios (Zhang y Zhou, 2018; Zhang et al., 2015). Una mayor disponibilidad de árboles se correspondió con el mayor uso de los UGS, siendo esta observación similar a la hallada por Vich et al. (2019b), mientras que, en el caso de las praderas, con mucha menos presencia, se correspondieron bajos porcentajes de tiempo empleado. Este comportamiento observado puede ser respaldado por el hecho de que no solo la oferta disponible influye en las decisiones del uso, sino también la calidad de dichas áreas, como afirman Van Herzele \& Wiedemann (2003).

Finalmente, prima un comportamiento sedentario total frente al activo, superándolo casi con el doble de valor y siendo próximo al valor correspondiente para el tiempo total. El predominio del comportamiento sedentario entre los participantes de la tercera edad ha sido confirmado por un estudio similar, corroborando las preferencias de las personas mayores por sentarse en bancos como la razón principal de su actividad sedentaria (Thompson et al., 2013). En el presente estudio, la edad de los participantes se ha asociado con un tiempo más sedentario. En esta línea, otros estudios confirmaron que las edades más jóvenes se asocian mayores tiempos físicamente activos, por lo que esta característica individual como es la edad puede resultar de gran importancia junto a la diversidad del paisaje a la hora de pasar un mayor tiempo en UGS (Keadle et al., 2016; Sparling et al., 2015).

\section{Limitaciones}

Este trabajo no está exento de limitaciones. En primer lugar, los participantes pueden haber tenido una conducta al caminar diferente a la habitual por el mero hecho de estar involucrados en este estudio. Del mismo modo, otra limitación es el hecho de que los participantes usaron los dispositivos en sus muñecas, lo que podría haber generado que ciertos momentos de actividad no se hubieran registrado debido a la falta de movimiento de los brazos. El análisis de este estudio podría ser parcial con los participantes interesados en que el estudio presente un buen estado de salud, mejor que el de la población mayor promedio. En consecuencia, la selección de los centros para personas mayores participantes se seleccionó al azar y no siguió ningún esquema de muestreo preestablecido. Adicionalmente, para este estudio no se tuvieron en cuenta la influencia de ciertos elementos tales como bancos, fuentes potables o aseos en el tipo de actividades y tiempo total de los UGS analizados.

\section{Conclusiones}

El presente estudio tuvo como objetivo investigar las relaciones entre la provisión de diferentes tipos de vegetación y superficies transitables disponibles en espacios verdes urbanos (UGS) con la intensidad de las actividades realizadas por los ancianos. En este trabajo, la influencia de los atributos de UGS en el comportamiento de los participantes mayores se midió mediante el uso de información SIG disponible públicamente y se combinó con datos de GPS y acelerómetro.

Los resultados de este estudio generan nuevos conocimientos sobre la planificación y gestión de UGS en cuanto a su uso por parte de las personas mayores. En esta línea, la edad de la población potencial es un aspecto clave para tener en cuenta al diseñar y construir UGS que sean inclusivos y accesibles para todos los perfiles de la sociedad. Además, el tipo de superficie transitable ha demostrado jugar un papel relevante con los diferentes comportamientos de actividad física cuando se trata de pasar más tiempo en estos espacios. 


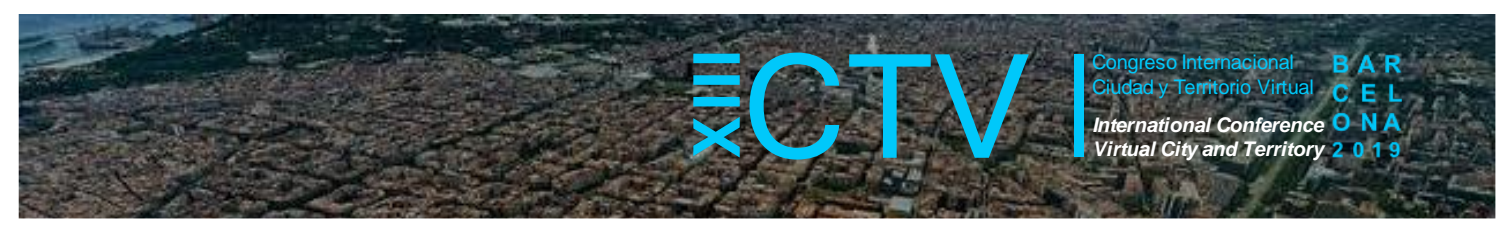

De este modo, las superficies duras se han relacionado con un mayor tiempo, mientras que, por el contrario, las superficies blandas y mixtas implican un menor tiempo en los UGS.

Basándose en estos resultados, el acopio de infraestructuras en UGS debe centrarse en fomentar el comportamiento sedentario y activo para motivar los usos. Asimismo, la existencia de sombras, bancos y superficies bien mantenidas y definidas son algunos de los elementos que se deberían considerar al diseñar UGS para personas mayores.

Contribuciones de los autores: Los autores han trabajado de manera conjunta.

Conflicto de Intereses: Los autores declaran que no hay conflicto de intereses.

\section{Bibliografía}

Ajuntament de Barcelona. (2018a). Plan del Verde y de la Biodiversidad de Barcelona 2020, 2013. Recuperado de https://ajuntament.barcelona.cat/ecologiaurbana/ca

Ajuntament de Barcelona. (2018b). Statistical Yearbook of Barcelona City. Year 2018. Recuperado de http://www.bcn.cat/estadistica/angles/dades/anuari/

Ajuntament de Barcelona. (2018c). Plan del Verde y de la Biodiversidad de Barcelona 2020, 2013. Recuperado de https://ajuntament.barcelona.cat/ecologiaurbana/ca

Ajuntament de Barcelona. (2016). Land Use Map from Barcelona City Council. Recuperado de http://w20.bcn.cat/cartobcn/

Ambiente Italia Research Institute. 2003. European Common Indicators: Towards a Local Sustainability Profile. Final Report. Milano, Italy.

Arnberger, A., \& Eder, R. (2011). The influence of age on recreational trail preferences of urban green-space visitors: a discrete choice experiment with digitally calibrated images. Journal of Environmental Planning and Management, 54(7), 891-908.

Arnberger, A., \& Eder, R. (2015). Are urban visitors' general preferences for green-spaces similar to their preferences when seeking stress relief? Urban Forestry \& Urban Greening, 14(4), 872-882.

Baran, P. K., Smith, W. R., Moore, R. C., Floyd, M. F., Bocarro, J. N., Cosco, N. G., \& Danninger, T. M. (2014). Park use among youth and adults: examination of individual, social, and urban form factors. Environment and Behavior, 46(6), 768-800.

Barnett, D. W., Barnett, A., Nathan, A., Van Cauwenberg, J., \& Cerin, E. (2017). Built environmental correlates of older adults' total physical activity and walking: a systematic review and meta-analysis. International Journal of Behavioral Nutrition and Physical Activity, 14(1), 103.

Bauman, A. E., Reis, R. S., Sallis, J. F., Wells, J. C., Loos, R. J., Martin, B. W., \& Lancet Physical Activity Series Working Group. (2012). Correlates of physical activity: why are some people physically active and others not? The lancet, 380(9838), 258-271. 


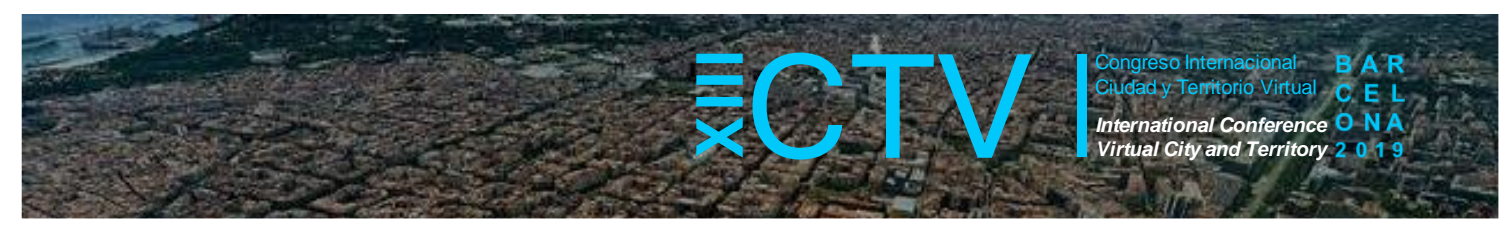

Besenyi, G. M., Kaczynski, A. T., Stanis, S. A. W., \& Vaughan, K. B. (2013). Demographic variations in observed energy expenditure across park activity areas. Preventive medicine, 56(1), 79-81. Biol. Lett., 5 (3) (2009), pp. 352-355.

Chiang, Y. C., \& Li, D. (2019). Metric or topological proximity? The associations among proximity to parks, the frequency of residents' visits to parks, and perceived stress. Urban forestry \& urban greening, 38, 205-214.

Costigan, S., Veitch, J., Crawford, D., Carver, A., \& Timperio, A. (2017). A cross-sectional investigation of the importance of park features for promoting regular physical activity in parks. International journal of environmental research and public health, 14(11), 1335.

Daniels, B., Zaunbrecher, B. S., Paas, B., Ottermanns, R., Ziefle, M., \& Roß-Nickoll, M. (2018). Assessment of urban green space structures and their quality from a multidimensional perspective. Science of the Total Environment, 615, 1364-1378.

Esliger, D. W., Rowlands, A. V., Hurst, T. L., Catt, M., Murray, P. \& Eston, R. G. Validation of the GENEA accelerometer. Medicine and Science in Sports and Exercise 2011, 43(6), 1085-93.

Fuller, R. A., \& Gaston, K. J. (2009). The scaling of green space coverage in European cities. Biology letters, 5(3), 352-355.

Gaikwad, A., \& Shinde, K. (2019). Use of parks by older persons and perceived health benefits: A developing country context. Cities, 84, 134-142.

Heiland, E. G., Welmer, A. K., Wang, R., Santoni, G., Fratiglioni, L., \& Qiu, C. (2019). Cardiovascular risk factors and the risk of disability in older adults: variation by age and functional status. Journal of the American Medical Directors Association, 20(2), 208-212.

Hoyle, H., Hitchmough, J., \& Jorgensen, A. (2017). All about the 'wow factor'? The relationships between aesthetics, restorative effect and perceived biodiversity in designed urban planting. Landscape and Urban Planning, 164, 109-123.

Jankowska, M. M., Schipperijn, J., \& Kerr, J. (2015). A framework for using GPS data in physical activity and sedentary behavior studies. Exercise and sport sciences reviews, 43(1), 48.

Jansen, F. M., Ettema, D. F., Kamphuis, C. B. M., Pierik, F. H., \& Dijst, M. J. (2017). How do type and size of natural environments relate to physical activity behavior? Health \& place, 46, 73-81.

Kabisch, N., Frantzeskaki, N., Pauleit, S., Naumann, S., Davis, M., Artmann, M. \& Zaunberger, K. (2016). Nature-based solutions to climate change mitigation and adaptation in urban areas: perspectives on indicators, knowledge gaps, barriers, and opportunities for action. Ecology and Society, 21(2)39. 


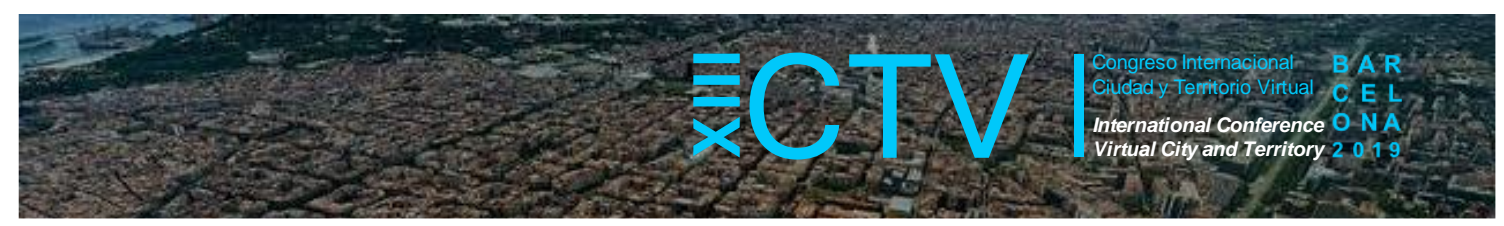

Keadle, S. K., McKinnon, R., Graubard, B. I., \& Troiano, R. P. (2016). Prevalence and trends in physical activity among older adults in the United States: a comparison across three national surveys. Preventive medicine, 89, 37-43.

Lindgren, T. \& Nilsen, M. R. (2012). Safety in residential areas. Tijdschrift voor economische en sociale geografie, 103(2), 196-208.

Livesley, S. J., McPherson, E. G., \& Calfapietra, C. (2016). The urban forest and ecosystem services: impacts on urban water, heat, and pollution cycles at the tree, street, and city scale. Journal of environmental quality, 45(1), 119-124.

Margaritis, E., \& Kang, J. (2017). Relationship between green space-related morphology and noise pollution. Ecological indicators, 72, 921-933.

Marquet, O., Hipp, J. A., Alberico, C., Huang, J. H., Fry, D., Mazak, E., ... \& Floyd, M. F. (2019). Park use preferences and physical activity among ethnic minority children in low-income neighborhoods in New York City. Urban forestry \& urban greening, 38, 346-353.

Natural England. 2010. «Nature Nearby» Accessible Natural Greenspace Guidance.

Recchioni, R., Marcheselli, F., Antonicelli, R., Mensa, E., Lazzarini, R., Procopio, A. D., \& Olivieri, F. (2017). Epigenetic effects of physical activity in elderly patients with cardiovascular disease. Experimental gerontology, 100, 17-27.

Reis, R. S., Hino, A. A. F., Florindo, A. A., Añez, C. R. R., \& Domingues, M. R. (2009). Association between physical activity in parks and perceived environment: a study with adolescents. Journal of Physical Activity and Health, 6(4), 503-509.

Rodiek, S. D., \& Fried, J. T. (2005). Access to the outdoors: Using photographic comparison to assess preferences of assisted living residents. Landscape and urban planning, 73(2-3), 184199.

Roe, J., Thompson, C., Aspinall, P., Brewer, M., Duff, E., Miller, D., ... \& Clow, A. (2013). Green space and stress: evidence from cortisol measures in deprived urban communities. International journal of environmental research and public health, 10(9), 4086-4103.

Schipperijn, J., Bentsen, P., Troelsen, J., Toftager, M., \& Stigsdotter, U. K. (2013). Associations between physical activity and characteristics of urban green space. Urban Forestry \& Urban Greening, 12(1), 109-116.

Shores, K. A., West, S. T., Evans, A. W., \& Evans, D. (2006, October). The relative importance of social and environmental attributes for active park use. In the Cooper Institute Scientific Conference Series: Parks, Recreation, and Public Health: Collaborative Frameworks for Promoting Physical Activity.

Sparling, P. B., Howard, B. J., Dunstan, D. W., \& Owen, N. (2015). Recommendations for physical activity in older adults. Bmj, 350, h100. 


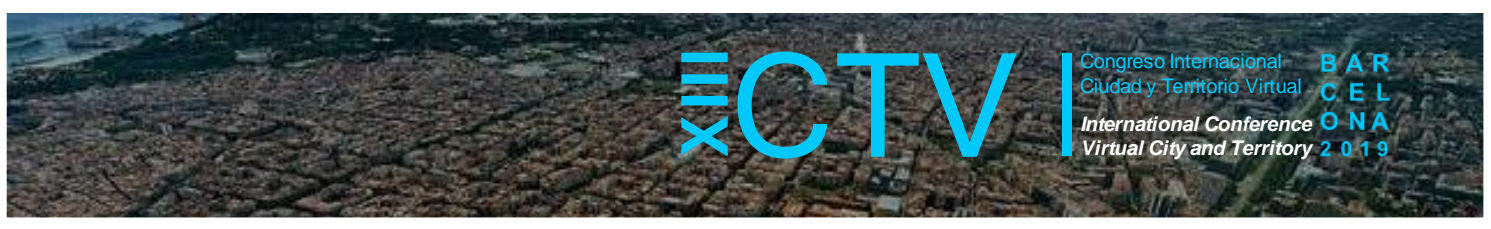

Stewart, O. T., Moudon, A. V., Fesinmeyer, M. D., Zhou, C., \& Saelens, B. E. (2016). The association between park visitation and physical activity measured with accelerometer, GPS, and travel diary. Health \& place, 38, 82-88.

Sugiyama, T., \& Thompson, C. W. (2008). Associations between characteristics of neighbourhood open space and older people's walking. Urban forestry \& urban greening, 7(1), 41-51.

Van Herzele, A., \& Wiedemann, T. (2003). A monitoring tool for the provision of accessible and attractive urban green spaces. Landscape and urban planning, 63(2), 109-126.

Veitch, J., Salmon, J., Deforche, B., Ghekiere, A., Van Cauwenberg, J., Bangay, S., \& Timperio, A. (2017). Park attributes that encourage park visitation among adolescents: A conjoint analysis. Landscape and urban planning, 161, 52-58.

Vich, G., Marquet, O., \& Miralles-Guasch, C. (2019a). Green exposure of walking routes and residential areas using smartphone tracking data and GIS in a Mediterranean city. Urban Forestry \& Urban Greening, 40, 275-285.

Vich, G., Marquet, O., \& Miralles-Guasch, C. (2019b). Green streetscape and walking: exploring active mobility patterns in dense and compact cities. Journal of Transport \& Health, 12, 50-59.

Wang, R., Zhao, J., Meitner, M. J., Hu, Y., \& Xu, X. (2019). Characteristics of urban green spaces in relation to aesthetic preference and stress recovery. Urban Forestry \& Urban Greening, 41, 6-13.

White, M., Smith, A., Humphryes, K., Pahl, S., Snelling, D., \& Depledge, M. (2010). Blue space: The importance of water for preference, affect, and restorativeness ratings of natural and built scenes. Journal of Environmental Psychology, 30(4), 482-493.

WHO. 2016. Urban green spaces and health: a review of the evidence. Copenhagen.

Wu, K. C., \& Song, L. Y. (2017). A case for inclusive design: analyzing the needs of those who frequent Taiwan's urban parks. Applied ergonomics, 58, 254-264.

Zhai, Y., \& Baran, P. K. (2017). Urban park pathway design characteristics and senior walking behavior. Urban forestry \& urban greening, 21, 60-73.

Zhai, Y., Baran, P. K., \& Wu, C. (2018). Spatial distributions and use patterns of user groups in urban forest parks: An examination utilizing GPS tracker. Urban forestry \& urban greening, 35, $32-44$

Zhang, S., \& Zhou, W. (2018). Recreational visits to urban parks and factors affecting park visits: Evidence from geotagged social media data. Landscape and urban planning, 180, 27-35.

Zhang, W., Yang, J., Ma, L., \& Huang, C. (2015). Factors affecting the use of urban green spaces for physical activities: Views of young urban residents in Beijing. Urban Forestry \& Urban Greening, 14(4), 851-857. 